\title{
Length spectra and the Teichmüller metric for surfaces with boundary
}

\author{
Lixin Liu • Athanase Papadopoulos • Weixu Su • \\ Guillaume Théret
}

Received: 13 April 2009 / Accepted: 26 May 2009 / Published online: 5 August 2009 (C) The Author(s) 2009. This article is published with open access at Springerlink.com

\begin{abstract}
We consider some metrics and weak metrics defined on the Teichmüller space of a surface of finite type with nonempty boundary, that are defined using the hyperbolic length spectrum of simple closed curves and of properly embedded arcs, and we compare these metrics and weak metrics with the Teichmüller metric. The comparison is on subsets of Teichmüller space which we call " $\varepsilon_{0}$-relative $\epsilon$-thick parts", and whose definition depends on the choice of some positive constants $\varepsilon_{0}$ and $\epsilon$. Meanwhile, we give a formula for the Teichmüller metric of a surface with boundary in terms of extremal lengths of families of arcs.
\end{abstract}

Keywords Riemann surface with boundary - Teichmüller space $\cdot$ Teichmüller metric $\cdot$ Length spectrum metric $\cdot$ Length spectrum weak metrics $\cdot$ Extremal length

Lixin Liu and Weixu Su were partially supported by NSFC (No. 10871211).

L. Liu . W. Su

Department of Mathematics, Zhongshan University,

510275 Guangzhou, Peoples's Republic of China

e-mail: mcsllx@mail.sysu.edu.cn

W. Su

e-mail: su023411040@163.com

A. Papadopoulos · G. Théret

Max-Plank-Institut für Mathematik, Vivatsgasse 7, 53111 Bonn, Germany

e-mail: theret@mpim-bonn.mpg.de

A. Papadopoulos $(\varangle)$

Institut de Recherche Mathématique Avancée, Université de Strasbourg and CNRS,

7 rue René Descartes, 67084 Strasbourg Cedex, France

e-mail: papadopoulos@math.u-strasbg.fr 
Mathematics Subject Classification (2000) $\quad 32 \mathrm{G} 15 \cdot 30 \mathrm{~F} 30 \cdot 30 \mathrm{~F} 60$

\section{Contents}

1 Introduction . . . . . . . . . . . . . . . . . . . . . . . . . . 296

2 On the geometry of surfaces with boundary . . . . . . . . . . . . . . . . . 297

3 Extremal length and the Teichmüller metric . . . . . . . . . . . . . . . 300

4 The relative thick part of Teichmüller space and Mumford's compactness theorem . . . . . . 305

5 Length spectrum weak metrics . . . . . . . . . . . . . . . . . . . . . . . 306

6 Comparison of length spectrum metrics with the Teichmüller metric . . . . . . . . . . . 308

\section{Introduction}

In this paper, $S$ is a connected oriented surface of finite topological type with nonempty boundary. The surface $S$ is obtained from a closed oriented surface by removing a finite number $p \geq 0$ of punctures and a finite number $b \geq 1$ of disjoint open disks. We shall say that $S$ has $p$ punctures and $b$ boundary components, or that it is of type $(g, p, b)$. The boundary of $S$ is denoted by $\partial S$, and we shall assume throughout the paper that $\partial S \neq \emptyset$ unless the contrary is explicitly stated. The Euler characteristic of $S$ is equal to $\chi(S)=2-2 g-p-b$, and we shall always assume that $\chi(S)<0$.

We shall consider conformal (or Riemann surface) structures on $S$. By the expression "conformal structure" on a surface $S$ with nonempty boundary, we mean that the topological interior of $S$ is equipped with a conformal structure in the usual sense, and that the following two properties are satisfied:

(1) each puncture has a neighborhood which is conformally equivalent to the punctured disk $\{z \in \mathbb{C}|0<| z \mid<1\}$;

(2) each boundary component has a neighborhood which is conformally equivalent to an annulus of finite modulus $\{z \in \mathbb{C}|a \leq| z \mid<1\}$ for some positive constant $a$ that depends on the puncture.

To simplify notation, we shall also denote by the same letter $S$ the surface equipped with a conformal structure. Then, $\bar{S}$ denotes the surface $S$ equipped with the mirror-image conformal structure. We recall that this means that a conformal atlas for $\bar{S}$ is obtained from an atlas for $S$ by composing each local coordinate $z$ for $S$ with the conjugation map $z \mapsto \bar{z}$. We let $S^{d}=S \cup \bar{S}$ denote the conformal double of $S$, obtained by gluing $S$ and $\bar{S}$ along corresponding boundary components, using the identity map. The surface $S^{d}$ is equipped with a natural anti-holomorphic involution, whose fixed point set is the boundary of $S$, considered as embedded in $S^{d}$. By the Poincaré uniformization theorem, $S^{d}$ carries a unique complete hyperbolic metric which is compatible with its conformal structure. Because of the symmetry in the definition of $\bar{S}$, this hyperbolic metric is invariant under the natural anti-holomorphic involution of $S^{d}$. The canonical hyperbolic metric on $S$ is the restriction to $S$ (with respect to the natural inclusion $S \subset S^{d}$ ) of this complete hyperbolic metric on $S^{d}$. The conformal structure that underlies the canonical hyperbolic metric on $S$ is the conformal structure that we started with. 
We denote by $\mathcal{T}(S)$ the reduced Teichmüller space of marked conformal structures on $S$. This is the set of equivalence classes of pairs $(X, f)$, where $X$ is a Riemann surface and $f: S \rightarrow X$ is a homeomorphism (called the marking), and where $\left(X_{1}, f_{1}\right)$ is considered to be equivalent to $\left(X_{2}, f_{2}\right)$ if there is a conformal map $h: X_{1} \rightarrow X_{2}$ which is homotopic to $f_{2} \circ f_{1}^{-1}$. We recall that in the reduced theory, homotopies need not fix the boundary of $S$ pointwise. Since all Teichmüller spaces that we consider are reduced, we shall omit the word "reduced" in our exposition. Furthermore, we shall often denote an element $(X, f)$ of $\mathcal{T}(S)$ by $X$, without explicit reference to the marking.

The elements of $\mathcal{T}(S)$ can also be taken to be (equivalence classes of) hyperbolic metrics with geodesic boundary, and these metrics will always be the canonical hyperbolic metrics for the conformal structures they induce.

In the paper [9], we studied some metrics and weak metrics on the Teichmüller space $\mathcal{T}(S)$, defined using the hyperbolic length spectrum of simple closed curves and of properly embedded arcs in $S$. In the present paper, we compare these metrics and weak metrics with the Teichmüller metric on $\mathcal{T}(S)$, on subsets of this space which we call " $\varepsilon_{0}$-relative $\epsilon$-thick parts", whose definition depends on the choice of some positive constants $\varepsilon_{0}$ and $\epsilon$, and which we describe below.

\section{On the geometry of surfaces with boundary}

We need to give some complements to Thurston's theory concerning a surface $S$ with boundary. As a general rule, the point is to include in the theory the study of arcs joining boundary components, instead of dealing only with simple closed curves on the surface. As we shall see, many results on spaces associated to a surface $S$ with boundary can be recovered from corresponding results on a surface without boundary by taking the double of $S$. In this section, we recall some definitions and give precise statements concerning some topological and conformal notions on surfaces with boundary. Abikoff's book [1] deals with surfaces with boundary, and there is also a small subsection on quadratic differentials on surfaces with boundary in Strebel's book (see [18, p. 157]).

We first recall a few topological definitions. A simple closed curve in $S$ is said to be peripheral if it is homotopic to a puncture. It is said to be essential if it is nonperipheral and if it is not homotopic to a point (but it can be homotopic to a boundary component).

We let $\mathrm{C}=\mathrm{C}(S)$ be the set of homotopy classes of essential simple closed curves on $S$.

An $\operatorname{arc}$ in $S$ is the homeomorphic image of a closed interval which is properly embedded in $S$ (that is, the interior of the arc is in the interior of $S$ and the endpoints of the arc are on the boundary of $S$ ). All homotopies of arcs that we consider are relative to $\partial S$, that is, they leave the endpoints of arcs on the set $\partial S$ (but they do not necessarily fix pointwise the endpoints). An arc is said to be essential if it is not homotopic (relative to $\partial S$ ) to a map whose image is in $\partial S$.

We let $\mathcal{B}=\mathcal{B}(S)$ be the union of the set of homotopy classes of essential arcs on $S$ with the set of homotopy classes of simple closed curves which are homotopic to boundary components. 
We shall denote by $[\gamma] \in \mathcal{B} \cup \mathcal{C}$ the equivalence class of a simple closed curve or of an $\operatorname{arc} \gamma$ on $S$.

For any $[\gamma] \in \mathcal{B} \cup \mathcal{C}$ and for any $X \in \mathcal{T}(S)$, we let $\gamma^{X}$ be the geodesic representative of $[\gamma]$ (that is, the curve of shortest length in the homotopy class relative to $\partial S$ ) with respect to the hyperbolic structure $X$, if $X$ is a hyperbolic structure, or with respect to the canonical hyperbolic metric associated to the conformal structure $X$, if $X$ is only a conformal structure. The geodesic $\gamma^{X}$ is unique; it coincides with a boundary component if $[\gamma]$ is the homotopy class of that boundary component, and it is orthogonal to $\partial S$ at each intersection point if $[\gamma]$ is the homotopy class of an arc. We denote by $l_{X}(\gamma)$ the length of $\gamma^{X}$ with respect to the hyperbolic metric $X$. To simplify notation, we shall sometimes denote $[\gamma]$ by $\gamma$.

We denote by $\mathcal{M} \mathcal{L}(S)$ the space of measured geodesic laminations on $S$. This space is equipped with a topology defined by Thurston (cf. [19]).

We recall that any measured geodesic lamination on a hyperbolic surface of finite topological type can be decomposed as the union of finitely many components whose support is of one of the following three types:

(1) an essential geodesic arc;

(2) a boundary component;

(3) a geodesic lamination in the interior of $S$ which is minimal, that is, in which every leaf is dense.

Finally, we recall that there are natural homeomorphisms between the spaces $\mathcal{M} \mathcal{L}(S)$, when the hyperbolic structure on $S$ varies, so that it is possible to talk about a measured geodesic laminations on $S$ without referring to a specific hyperbolic structure on that surface. Such a space $\mathcal{M} \mathcal{L}(S)$ is also canonically homeomorphic to the space $\mathcal{N} \mathcal{F}(S)$ of equivalence classes of measured foliations on $S$. An arc (respectively, hyperbolic structure, simple closed curve, measured lamination, etc.) on $S^{d}$ is said to be symmetric if it is invariant by the natural involution.

In what follows, quadratic differentials are as usual assumed to be meromorphic with at most simple poles at punctures. Quadratic differentials on a conformal surface with boundary are assumed to take real values on vectors tangent to the boundary components. More precisely, such a quadratic differential $\phi$ can have (isolated) zeroes on $\partial S$, and it satisfies $\phi(z) d z^{2}>0$ or $\phi(z) d z^{2}<0$ for any tangent vector $d z$ which is tangent to $\partial S$ at a point on $\partial S$ which is in the complement of the zeroes of $\phi$.

A quadratic differential equips the underlying surface with a pair of measured foliations, namely, its horizontal and vertical foliations. On surfaces with boundary, measured foliations are allowed to be transverse to the boundary or tangent to the boundary, and both cases can occur at the same boundary component, as in Fig. 1. By a result of Hubbard and Masur (cf. [7], adapted to the case of surfaces with boundary), the ordered pair consisting of the horizontal and vertical measured foliation of a quadratic differential (and even, the pair of their Whitehead equivalence classes in the sense of Thurston) completely determines this quadratic differential.

Given a surface $S$ equipped with a quadratic differential $\phi$, each point on a boundary component of $S$ which is a nonzero point of $\phi$ has a neighborhood where the horizontal and vertical measured foliations are either perpendicular or tangent to the boundary. (As already said, both kinds of behavior can occur at a given boundary component, as 


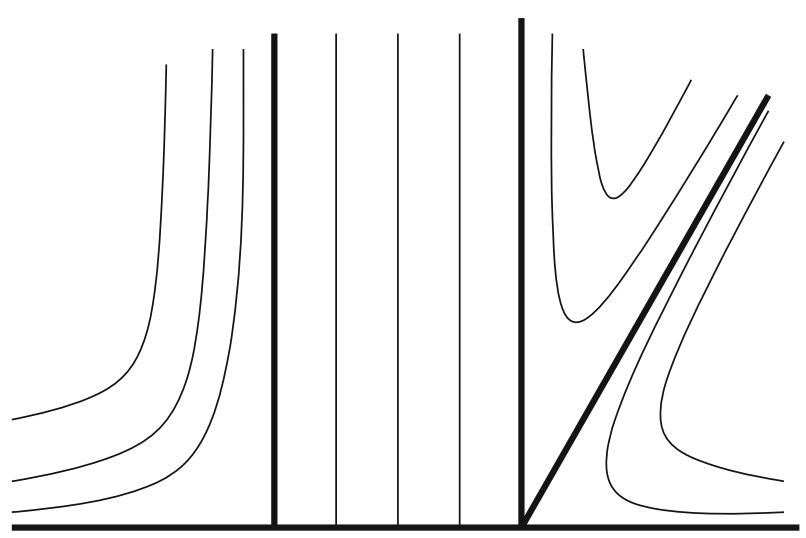

Fig. 1 The leaves of a measured foliation can be either transverse or tangent to the boundary component of the surface

in Fig. 1). The quadratic differential $\phi$ gives, by reflection along $\partial S$, a well-defined quadratic differential on the double $S^{d}$ of $S$.

The Teichmüller metric $d_{T}$ on $\mathcal{T}(S)$ is defined by

$$
d_{T}(X, Y)=\frac{1}{2} \inf \log K(f)
$$

for any two marked conformal structures $X$ and $Y$ on $S$, where the infimum is taken over all quasiconformal homeomorphisms $f: X \rightarrow Y$ and where $K(f)$ is the dilatation of $f$.

If $X$ and $Y$ are two conformal structures on $S$, then a quasiconformal homeomorphism $f: X \rightarrow Y$ extends to a quasiconformal homeomorphism $f^{d}: X^{d} \rightarrow Y^{d}$ between the conformal doubles, with the same dilatation $K(f)$.

A Teichmüller map $f: X \rightarrow Y$ extends to a Teichmüller map $f^{d}: X^{d} \rightarrow Y^{d}$. From the uniqueness of Teichmüller maps, $f^{d}$ commutes with the natural anti-holomorphic involutions of the Riemann surfaces $X^{d}$ and $Y^{d}$, and its associated initial quadratic differential is the double of the quadratic differential of $f$. In particular, the horizontal and vertical measured foliations of $f^{d}$ are symmetric with respect to the natural involution of $X^{d}$.

Theorem 2.1 (Wolpert's inequality for surfaces with boundary) Let $X$ and $Y$ be two hyperbolic structures on $S$ and let $f: X \rightarrow Y$ be a $K$-quasiconformal homeomorphism. For any element $\gamma$ which is either an essential simple closed curve or an essential arc on $S$, we have

$$
\frac{l_{Y}(f(\gamma))}{l_{X}(\gamma)} \leq K .
$$

The result for closed curves on surfaces without boundary is in Wolpert [21]. The result for arcs follows by doubling. Indeed, doubling a quasiconformal homeomorphism produces on the doubled surface a quasiconformal homeomorphism with the 
same dilatation. Doubling a geodesic $X$ - or $Y$-arc produces a simple closed geodesic on the doubled hyperbolic surface $X^{d}$ or $Y^{d}$ respectively. Wolpert's inequality for arcs in $S$ follows then from the inequality for simple closed curves on the double.

\section{Extremal length and the Teichmüller metric}

The notion of extremal length for families of curves or of arcs on a Riemann surface was introduced by Ahlfors and Beurling [2]. We follow the exposition that Kerckhoff gives in [8] for elements of $\mathcal{C}(S)$, which we adapt to elements of $\mathcal{B}(S) \cup \mathcal{C}(S)$.

There are two equivalent definitions of extremal length, one which Kerckhoff calls analytic, and another one which he calls geometric.

We consider a fixed Riemann surface structure on $S$. A conformal metric on $S$ is a Riemannian metric which may have isolated singularities and whose length element in every holomorphic coordinate $z$ in the complement of the singularities can be written as $\rho(z)|d z|$, where $\rho$ is a nonnegative real-valued function. We let $A_{X}$ denote the total area of the metric $X$.

Definition 3.1 (Analytic) The extremal length of $\gamma \in \mathcal{C}(S) \cup \mathcal{B}(S)$ is the quantity

$$
E_{X}(\gamma)=\sup _{X} \frac{l_{X}^{2}(\gamma)}{A_{X}}
$$

where the supremum is taken over all conformal metrics satisfying $0<A_{X}<\infty$.

Given a conformal metric $X$ on $S$, we shall also use the notation $E_{X}(\gamma)$ to denote the extremal length of a family $\gamma$ of closed curves or of arcs on $S$, meaning that we consider the extremal length of $\gamma$ with respect to the conformal structure underlying $X$. In general, the metrics $X$ that we shall consider will be hyperbolic.

Since the area of any hyperbolic metric $X$ on $S$ is equal to $2 \pi|\chi(S)|$, it follows from the above definition that for any element $\gamma$ in $\mathcal{B} \cup \mathcal{C}$ we have

$$
E_{X}(\gamma) \geq \frac{l_{X}^{2}(\gamma)}{2 \pi|\chi(S)|} .
$$

For the geometric definition of conformal length, we need to consider cylinders and quadrilaterals in Riemann surfaces, and we recall the definitions.

A cylinder in a Riemann surface $S$ is a surface with boundary which is homeomorphic to a compact Euclidean annulus, which is immersed in $S$ in such a way that its interior is embedded. If $C$ is any closed curve in $S$ homotopic to a boundary component of a cylinder, then we say that $C$ is a core curve of the cylinder.

We shall consider quadrilaterals only on Riemann surfaces with nonempty boundary. A quadrilateral in such a surface $S$ is a closed disk with two disjoint distinguished curves on its boundary, the disk being immersed in $S$ with embedded interior, such that the distinguished curves are arcs in $S$ in the sense defined above, and such that the two curves in the complement of the distinguished curves in the boundary of the quadrilateral are contained in $\partial S$. Note that the two distinguished curves are then homotopic in $S$ relative to $\partial S$. The distinguished curves and the two components of their complement in the boundary of the quadrilateral are called the edges of the quadrilateral. 
If $C$ is any arc in $S$ which is homotopic to one of the two distinguished edges of the quadrilateral, then we say that the quadrilateral has core arc $C$.

Any cylinder or quadrilateral in $S$ is equipped with a conformal structure induced from that of $S$. In this way, a cylinder in $S$ is conformally equivalent to a Euclidean annulus of the form $\left\{z \in \mathbb{C}, r_{1} \leq|z| \leq r_{2}\right\}$. Although such a Euclidean annulus is not unique, the quotient $r_{1} / r_{2}$ is well-defined up to the choice of the boundary component of the cylinder that is sent to the interior component of the Euclidean annulus, and it is called the modulus of the Euclidean annulus and of the given cylinder in $S$. (Sometimes a factor of $1 / 2 \pi$ is introduced in the definition of the modulus, but this is irrelevant to the present paper).

Likewise, any quadrilateral in $S$ is conformally equivalent to a Euclidean rectangle of the form $\left\{z \in \mathbb{C}, 0 \leq \Re(z) \leq r_{1}, 0 \leq \Im(z) \leq r_{2}\right\}$, with the distinguished edges sent to the vertical edges of this Euclidean rectangle. Again, although such a Euclidean rectangle is not unique, the quotient $r_{1} / r_{2}$ is uniquely defined, and it is called the modulus of the Euclidean rectangle and of the given quadrilateral in $S$.

The modulus of a quadrilateral or of an annulus $A$ in $S$ is denoted by $m(A)$.

Definition 3.2 (Geometric) The extremal length $E(\gamma)$ of an element $\gamma$ in $\mathcal{C}(S)$ (respectively in $\mathcal{B}(S)$ ) is equal to

$$
\inf _{A} \frac{1}{m(A)}
$$

where the infimum is taken over all cylinders (respectively quadrilaterals) in $S$ with core curve (respectively core arc) in the homotopy class $\gamma$.

We shall use the fact that the extremal length function has a continuous extension from the sets of homotopy classes of arcs and curves to the space of measured foliations. We refer to Kerckhoff [8] for the case of surfaces without boundary, and we note the following, concerning the definition of a measured foliation on a surface with boundary, which make the results apply for such surfaces:

A measured foliation on a surface $S$ with boundary is a measured foliation on the interior of $S$, which extends to the boundary of $S$, such that furthermore the following holds:

- The double of any measured foliation on $S$ is a measured foliation on $S^{d}$.

- The restriction to $S$ of any measured foliation on $S^{d}$ is a measured foliation on $S$. Here, the surface $S$ is considered as embedded in $S^{d}$ in such a way that its boundary $\partial S$ is in minimal position with respect to the foliation on $S^{d}$ (cf. [5, p. 76] for the notion of minimal position, called there "quasi-transverse" position).

Kerckhoff's formula for the Teichmüller metric in terms of extremal length is generalized as follows for surfaces with boundary:

Theorem 3.3 If $X$ and $Y$ are two conformal structures on $S$, then the Teichmüller distance between them is equal to

$$
d_{T}(X, Y)=\frac{1}{2} \log \sup _{\gamma \in \operatorname{CuB}} \frac{E_{Y}(\gamma)}{E_{X}(\gamma)} .
$$


Proof In the case of closed surfaces, the proof of this formula is in [8, p. 36] (with the supremum being taken over $\gamma$ in $\mathcal{C}$, since the set $\mathcal{B}$ is empty). For a surface of finite type without boundary, the same proof applies because the curves which are parallel to the punctures (that is, the peripheral curves), are not part of the set $\mathcal{C}$, and therefore they are not involved in the proof. For general surfaces of finite topological type, the formula is obtained by taking the double, and we now outline the proof.

We have $d_{T}(X, Y)=d_{T}\left(X^{d}, Y^{d}\right)$, since Teichmüller distances are realized by Teichmüller maps, and, as we recalled above, a Teichmüller map between the surfaces without boundary $X^{d}$ and $Y^{d}$ induces a map between $X$ and $Y$, with the same dilatation, which will also be the Teichmüller map between the two doubles, since Teichmüller maps are unique. (Note that we are using the same notation $d_{T}$ for the Teichmüller distance on $\mathcal{T}(S)$ and of $\left.\mathcal{T}\left(S^{d}\right)\right)$.

By Kerckhoff's formula for surfaces without boundary, we have

$$
d_{T}\left(X^{d}, Y^{d}\right)=\frac{1}{2} \log \sup _{\gamma \in \mathcal{C}\left(S^{d}\right)} \frac{E_{Y^{d}}(\gamma)}{E_{X^{d}}(\gamma)}
$$

and the supremum is realized by the extremal length of the vertical foliation of the initial quadratic differential $q$ associated to the Teichmüller map between the conformal structures $X$ and $Y$ (see also [8, p. 36]). Since the quadratic differential and its vertical foliation are invariant by the natural involution of $X^{d}$, and since the Teichmüller map restricts to a Teichmüller map between the two surfaces $X$ and $Y$, the supremum

$$
\sup _{\gamma \in \mathcal{C}(S) \cup \mathcal{B}(S)} \frac{E_{Y}(\gamma)}{E_{X}(\gamma)}
$$

is realized by the restriction on $X$ of the vertical foliation of the quadratic differential $q$. This implies

$$
d_{T}(X, Y) \leq \frac{1}{2} \log \sup _{\gamma \in \mathcal{C}(S) \cup \mathcal{B}(S)} \frac{E_{Y}(\gamma)}{E_{X}(\gamma)}
$$

But the last inequality must be an equality, since if there were an element $\gamma$ in $\mathrm{C}(S) \cup$ $\mathcal{B}(S)$ satisfying

$$
d_{T}(X, Y)<\frac{1}{2} \log \frac{E_{Y}(\gamma)}{E_{X}(\gamma)},
$$

then, taking the doubles of the Teichmüller maps between $X$ and $Y$ and the double $\gamma^{d}$ of $\gamma$, we would have an element $\gamma^{d}$ in $\mathcal{C}\left(S^{d}\right) \cup \mathcal{B}\left(S^{d}\right)$ satisfying

$$
d_{T}\left(X^{d}, Y^{d}\right)<\frac{1}{2} \log \frac{E_{Y}\left(\gamma^{d}\right)}{E_{X}\left(\gamma^{d}\right)},
$$

which by Kerckhoff's formula for the Teichmüller metric of surfaces without boundary is excluded. 
We now deduce from Theorem 3.3 another formula for the Teichmüller metric on the Teichmüller space of a surface with boundary, which instead of using the extremal lengths of closed curves and of arcs, uses only extremal lengths of arcs. The idea is to approximate curves with arcs. We start with the following lemma which is extracted from [9]:

Lemma 3.4 Let $\beta$ be a component of $\partial S$, let $\alpha$ be a measured foliation on $S$ and let $\alpha^{d}$ be its double. Then, there exists a sequence of symmetric simple closed curves on $S^{d}$ converging to $\alpha^{d}$ in the topology of $\mathcal{P} \mathcal{N} \mathcal{F}\left(S^{d}\right)$ such that each of these simple closed curves intersects essentially in exactly two points the image of $\beta$ in $S^{d}$, and intersects no other component of the image of $\partial S$ in $S^{d}$.

From this we deduce the following:

Theorem 3.5 If $X$ and $Y$ are two conformal structures on $S$, then the Teichmüller distance between them is equal to

$$
d_{T}(X, Y)=\frac{1}{2} \log \sup _{\mathcal{B}} \frac{E_{Y}(\gamma)}{E_{X}(\gamma)} .
$$

Proof We use the continuous extension of the extremal length function on the space $\mathcal{M} \mathcal{F}(S)$ of measured foliations. Let $\alpha$ be a simple closed curve on $S$. Consider its double $\alpha^{d}$ in $S^{d}$. By Lemma 3.4, there exists a sequence of symmetric weighted simple closed curves $\left(\gamma_{n}^{d}\right)$ such that $\lim _{n \rightarrow \infty} \gamma_{n}^{d}=\alpha^{d}$ in $\mathcal{M} \mathcal{F}(S)$. Let $X^{d}$ and $Y^{d}$ be the doubles of the conformal structures $X$ and $Y$. We can assume the sequence of symmetric simple closed curves $\left(\gamma_{n}^{d}\right)$ on $S^{d}$ to be equipped, as elements of $\mathcal{M F}\left(S^{d}\right)$, with the counting measure. Then, we have

$$
\left|\frac{E_{Y^{d}}\left(\gamma_{n}^{d}\right)}{E_{X^{d}}\left(\gamma_{n}^{d}\right)}-\frac{E_{Y^{d}}\left(\alpha^{d}\right)}{E_{X^{d}}\left(\alpha^{d}\right)}\right| \rightarrow 0 .
$$

We conclude that for any simple closed curve $\alpha$ in $S$, there exists a sequence of connected $\operatorname{arcs}\left(\gamma_{n}\right)$ in $\mathcal{B}$ such that

$$
\left|\frac{E_{Y}\left(\gamma_{n}\right)}{E_{X}\left(\gamma_{n}\right)}-\frac{E_{Y}(\alpha)}{E_{X}(\alpha)}\right| \rightarrow 0 .
$$

This gives

$$
\sup _{\alpha \in \mathcal{B} \cup \mathcal{C}} \frac{E_{Y}(\alpha)}{E_{X}(\alpha)}=\sup _{\gamma \in \mathcal{B}} \frac{E_{Y}(\gamma)}{E_{X}(\gamma)},
$$

which concludes the proof.

Let us study a little further the function

$$
(X, Y) \mapsto \frac{1}{2} \log \sup _{\mathcal{e}} \frac{E_{Y}(\gamma)}{E_{X}(\gamma)} .
$$


First note that, in the case where the surface $S$ is a pair of pants, this function does not define a metric, since it can take negative values. To see this, we take two conformal structures $X$ and $Y$ on $S$ such that for each of the structures, the three boundary components have the same extremal length (for instance, we can take two hyperbolic structures such that the three boundary components of each one have the same hyperbolic lengths) and such that the value of the extremal length for the conformal structure $Y$ is less than the corresponding value for the structure $X$. It is clear in this case that $\frac{1}{2} \log \sup _{\mathcal{C}} \frac{E_{Y}(\gamma)}{E_{X}(\gamma)}<0$. Actually, one has more generally the following result, whose proof follows.

Proposition 3.6 Let $X$ be a Riemann surface with boundary. Then there exists a Riemann surface structure $Y \neq X$ on the same topological surface such that

$$
\frac{1}{2} \log \sup _{\mathcal{e}} \frac{E_{Y}(\gamma)}{E_{X}(\gamma)} \leq 0 .
$$

In particular, this shows that the function $\frac{1}{2} \log \sup _{\mathcal{C}} \frac{E_{Y}(\gamma)}{E_{X}(\gamma)}$ on $\mathcal{T}(S)$ does not separate points.

Remark 3.7 In [16], Parlier proved an analogue of Proposition 3.6 in the case where extremal lengths are replaced by hyperbolic lengths.

Before proving this proposition, let us recall a few facts on Nielsen extensions.

Let $X$ be a Riemann surface with boundary. Consider the unique complete hyperbolic metric associated to the conformal structure on the interior of $X$ by the Uniformization Theorem. This hyperbolic metric is complete and there exists, for each boundary component $C_{i}, i=1, \ldots, b$, of $X$, a unique simple closed geodesic $C_{i}^{\prime}$ which is freely homotopic to $C_{i}$ in $S$. By cutting $X$ along the geodesics $C_{i}^{\prime}, i=1, \ldots, b$, we obtain a Riemann surface $X_{0}$ and $b$ funnels adjacent to $C_{i}^{\prime}, i=1, \ldots, b$. Here a funnel is a Riemann surface of type $(0,0,2)$, that is, homeomorphic to an annulus. The hyperbolic surface with totally geodesic boundary $X_{0} \subset X$ is called the Nielsen kernel of $X$, and $X$ is the Nielsen extension of $X_{0}$ (see [3]). The Nielsen kernel is a deformation retract of $X$ and can be viewed as embedded in $X$.

The proof of Proposition 3.6 uses the following:

Lemma 3.8 Let $X_{1}$ be the Nielsen extension of $X_{0}$. Then for any $\gamma \in \mathcal{C}, E_{X_{1}}(\gamma) \leq$ $E_{X_{0}}(\gamma)$.

Proof This is a consequence of a monotonicity property for extremal length, which follows trivially from the definition.

For any $X, Y$ in $\mathcal{T}(S)$, set

$$
T(X, Y)=\frac{1}{2} \log \sup _{\gamma \in \mathcal{C}} \frac{E_{Y}(\gamma)}{E_{X}(\gamma)}
$$

and

$$
\bar{T}(X, Y)=\frac{1}{2} \log \sup _{\gamma \in \mathcal{C}} \frac{E_{X}(\gamma)}{E_{Y}(\gamma)} .
$$


From Lemma 3.8, we readily get

$$
T\left(X, X_{n}\right)=\frac{1}{2} \log \sup _{\gamma \in \mathcal{C}} \frac{E_{X_{n}}(\gamma)}{E_{X}(\gamma)} \leq 0 .
$$

Let $X_{1}$ be the Nielsen extension of $X$ and let $X_{k+1}$ be the Nielsen extension of $X_{k}$, $k=1,2, \ldots$. In view of the canonical embeddings $X_{k} \hookrightarrow X_{k+1}$ one can define the Riemann surface $X_{\infty}=X_{1} \cup X_{2} \cup \cdots$ which is called the infinite Nielsen extension of $X$.

Bers [3] proved the following.

Lemma 3.9 (Bers) The infinite Nielsen extension $X_{\infty}$ is a Riemann surface of type $(g, p+b, 0)$.

Let us recall the following result of Maskit [13] (which is easily extended from the case without boundary to the case with boundary by taking doubles).

Proposition 3.10 (Maskit) Let X be a Riemann surface of finite topological type and let $\gamma \in$ C. We have

$$
\frac{l_{X}(\gamma)}{\pi} \leq E_{X}(\gamma) \leq \frac{l_{X}(\gamma)}{2} e^{\frac{l_{X}(\gamma)}{2}}
$$

From Lemma 3.9, we have $\lim _{n \rightarrow \infty} l_{X_{n}}\left(C_{i}^{\prime}\right)=0, i=1, \ldots, b$. Combining with Lemma 3.10, we obtain

$$
\lim _{n \rightarrow \infty} \bar{T}\left(X, X_{n}\right)=\lim _{n \rightarrow \infty} \frac{1}{2} \log \sup _{\gamma \in \mathcal{C}} \frac{E_{X}(\gamma)}{E_{X_{n}}(\gamma)}=\infty .
$$

We say that two functions $f$ and $g$ defined over $\mathcal{T}(S)$ are called $(\lambda, C)$-quasi-isometric if there exist constants $C \geq 0$ and $\lambda \geq 1$ such that, for any $X, Y \in \mathcal{T}(S)$,

$$
\frac{1}{\lambda} g(X, Y)-C \leq f(X, Y) \leq \lambda g(X, Y)+C
$$

From what precedes we get the following

Theorem 3.11 The functions $T$ and $\bar{T}$ are not quasi-isometric on $\mathcal{T}(S)$.

Question 3.12 • Let $T^{\prime}(X, Y)=\max (T(X, Y), \bar{T}(X, Y))$, for $X, Y \in \mathcal{T}(S)$. Is $T^{\prime}(X, Y)$ a metric on $\mathcal{T}(S)$ ?

- Does there exist $X, Y \in \mathcal{T}(S)$, such that $T(X, Y)<0$ ?

\section{The relative thick part of Teichmüller space and Mumford's compactness theorem}

The extended mapping class group of $S$, denoted by $\operatorname{MCG}(S)$, is the group of homotopy classes of homeomorphisms of this surface. This group acts naturally on 
the Teichmüller space $\mathcal{T}(S)$, and the quotient space by this action is the moduli space $\mathcal{M}(S)$ of $S$. Elements of $\mathcal{M}(S)$ are therefore homotopy classes of hyperbolic surfaces without marking.

For any given $\epsilon>0$, we call the $\epsilon$-thick part of moduli space the subset of $\mathcal{M}(S)$ consisting of homotopy classes of unmarked hyperbolic surfaces satisfying the following two conditions:

(a) The length of any simple closed geodesic is $\geq \epsilon$.

(b) The length of any geodesic arc is $\geq \epsilon$.

A theorem by Mumford [14] says that in the case of surfaces of finite topological type without boundary, the $\epsilon$-thick part of moduli space (which in this case is simply the subset of $\mathcal{M}(S)$ consisting of homotopy classes of surfaces satisfying Condition (1)) is compact. The proof of this theorem that is given in [6] can be adapted without difficulty to the case of hyperbolic surfaces with boundary, if we include Condition (2) on geodesic arcs joining boundary components. For future reference, we state this as follows:

Theorem 4.1 (Mumford's compactness theorem for surfaces with boundary) For any topologically finite type surface $S$ and for any $\epsilon>0$, the $\epsilon$-thick part of the moduli space of $S$ is compact.

In the paper [9], given two real numbers $\epsilon$ and $\varepsilon_{0}$ satisfying $0<\epsilon \leq \varepsilon_{0}$, we considered the subspace of the Teichmüller space $\mathcal{T}(S)$, which we called the $\varepsilon_{0}$-relative $\epsilon$-thick part of Teichmülller space, consisting of equivalence classes of hyperbolic metrics satisfying the following two conditions:

(a) The length of any element in $\mathcal{C}$ is $\geq \epsilon$.

(b) The length of any boundary component of $S$ is $\leq \varepsilon_{0}$.

We have the following

Proposition 4.2 For any real numbers $\epsilon$ and $\varepsilon_{0}$ satisfying $0<\epsilon \leq \varepsilon_{0}$, the natural image of the $\varepsilon_{0}$-relative $\epsilon$-thick part of Teichmülller space in the moduli space $\mathcal{M}(S)$ is contained in some $\epsilon^{\prime}$-thick part of moduli space, for some $\epsilon^{\prime}>0$ depending only on $\epsilon$ and $\varepsilon_{0}$.

Proof We showed in [9] (Lemma 3.4) that for all $X$ in the $\varepsilon_{0}$-relative $\epsilon$-thick part of $\mathcal{T}(S)$, to any geodesic arc $\beta$ on $S$, we can associate a simple closed geodesic $\alpha$ on $S$ such that the ratio $l_{X}(\beta) / l_{X}(\alpha)$ is bounded above and below by positive constants that depends only on $\epsilon$ and $\varepsilon_{0}$. In particular, if the length of the simple closed geodesic $\alpha$ is bounded below by $\epsilon$, the length of the $\operatorname{arc} \beta$ is also bounded below by a constant that depends only on $\epsilon$ and $\varepsilon_{0}$. This gives the desired result.

\section{Length spectrum weak metrics}

We recall that a weak metric on a set $M$ is a function $\delta: M \times M \rightarrow[0, \infty)$ satisfying

(a) for $x$ and $y$ in $M, \delta(x, y)=0 \Longleftrightarrow x=y$; 
(b) for all $x, y$ and $z$ in $M, \delta(x, y)+\delta(y, z) \geq \delta(x, z)$.

and we say that the weak metric $\delta$ is asymmetric if furthermore

(c) there exist $x$ and $y$ in $M$ satisfying $\delta(x, y) \neq \delta(y, x)$.

In the paper [9], we considered the following two functions on $\mathcal{T}(S) \times \mathcal{T}(S)$, where $X$ and $Y$ are represented by hyperbolic structures:

$$
\begin{aligned}
& d(X, Y)=\log \sup _{\alpha \in \mathcal{C} \cup \mathcal{B}} \frac{l_{Y}(\alpha)}{l_{X}(\alpha)}, \\
& \bar{d}(X, Y)=\log \sup _{\alpha \in \mathcal{C} \cup \mathcal{B}} \frac{l_{X}(\alpha)}{l_{Y}(\alpha)} .
\end{aligned}
$$

The functions in (2) and (3) are analogues, for surfaces with boundary, of asymmetric weak metrics introduced by Thurston in [20] for surfaces of finite type without boundary. We showed that these functions are asymmetric weak metrics on $\mathcal{T}(S)$ (Proposition 2.9 of [9], where the point is to prove the separation property). We also showed that these weak metrics can be expressed using suprema over the set $\mathcal{B}$ only. More precisely, we proved the following ([9] Proposition 2.12):

For every $X$ and $Y$ in $\mathcal{T}(S)$, we have

$$
d(X, Y)=\log \sup _{\gamma \in \mathcal{B}} \frac{l_{Y}(\gamma)}{l_{X}(\gamma)}
$$

We also proved the following equalities (Corollary 2.8 of [9])

$$
d(X, Y)=d\left(X^{d}, Y^{d}\right), \quad \bar{d}(X, Y)=\bar{d}\left(X^{d}, Y^{d}\right) .
$$

The equalities in (5) are useful for obtaining results on the weak metrics $d$ and $\bar{d}$ for a surface $S$ with boundary from results on the corresponding weak metrics on the double of $S$, which is a surface without boundary.

We also studied the following symmetrization of the weak metrics $d$ and $\bar{d}$ :

$$
\delta_{L}(X, Y)=\log \max \left(\sup _{\gamma \in \mathcal{C} \cup \mathcal{B}} \frac{l_{Y}(\gamma)}{l_{X}(\gamma)}, \sup _{\gamma \in \mathcal{C} \cup \mathcal{B}} \frac{l_{X}(\gamma)}{l_{Y}(\gamma)}\right) .
$$

which, by (4), can also be expressed as

$$
\delta_{L}(X, Y)=\log \max \left(\sup _{\gamma \in \mathcal{B}} \frac{l_{Y}(\gamma)}{l_{X}(\gamma)} \sup _{\gamma \in \mathcal{B}} \frac{l_{X}(\gamma)}{l_{Y}(\gamma)}\right)=\max (d(X, Y), \bar{d}(X, Y)) .
$$

From (5), we immediately deduce the following, for all $X, Y$ in $\mathcal{T}(S)$ :

$$
\delta_{L}(X, Y)=\delta_{L}\left(X^{d}, Y^{d}\right) .
$$


In the same paper [9], we also considered the following symmetric function on the product $\mathcal{T}(S) \times \mathcal{T}(S)$ :

$$
d_{L}(X, Y)=\log \max \left(\sup _{\gamma \in \mathcal{C}} \frac{l_{X}(\gamma)}{l_{Y}(\gamma)} \sup _{\gamma \in \mathcal{C}} \frac{l_{Y}(\alpha)}{l_{X}(\alpha)}\right) .
$$

For surfaces of finite type without boundary, the functions $\delta_{L}$ and $d_{L}$ obviously coincide, and they define the so-called length spectrum metric on Teichmüller space. This metric was originally considered by Sorvali [17], and it has been studied by several authors, see e.g. $[4,11,12]$.

For surfaces of finite topological type with nonempty boundary, $\delta_{L}$ and $d_{L}$ are distinct, but they both are metrics. In the paper [9], we gave a comparison between these metrics in the thick part of Teichmüller space.

Note the formal analogy between Kerckhoff's Formula adapted to surfaces with boundary (Theorem 3.3) and Version (2) of the length spectrum asymmetric metric. But since the apparently asymmetric formula in Theorem 3.3 gives a genuine metric, Kerckhoff's formula is rather to be compared with the symmetrized length spectrum metric (7). Our goal in the next section is to give a precise comparison between these two metrics.

\section{Comparison of length spectrum metrics with the Teichmüller metric}

In this section, we use the preceding results to derive for surfaces with boundary some results which are already known for surfaces without boundary.

The first result is an immediate consequence of Wolpert's inequality (Theorem 2.1 above):

Theorem 6.1 For any surface $S$ of topologically finite type and for any $X$ and $Y$ in $\mathcal{T}(S)$, we have $\delta_{L}(X, Y) \leq 2 d_{T}(X, Y)$.

The second result follows from a result obtained in [15] for surfaces without boundary.

Theorem 6.2 Let $S$ be a surface of topologically finite type. The weak metrics $d, \bar{d}$ and $\delta_{L}$ on $\mathcal{T}(S)$ are complete.

The result for the weak metrics $d$ and $\bar{d}$ follows from the relations (5) and the completeness of the corresponding weak metrics for the Teichmüller spaces of surfaces of finite type and without boundary (see [15]). The result for the metric $\delta_{L}$ follows from the definition, $\delta_{L}=\max (d, \bar{d})$.

The following is an adaptation of the result obtained by Choi and Rafi in [4] for surfaces with boundary.

Theorem 6.3 Let $S$ be a surface of topologically finite type. For any $\epsilon$ and $\varepsilon_{0}$ satisfying $\varepsilon_{0} \geq \epsilon>0$, there is a constant $D$ depending on $\epsilon$ and $\varepsilon_{0}$ such that for any $X$ and $Y$ in the $\varepsilon_{0}$-relative $\epsilon$-thick part of $\mathcal{T}(S)$, we have 


$$
\begin{gathered}
d(X, Y)-D \leq d_{T}(X, Y) \leq d(X, Y)+D, \\
\bar{d}(X, Y)-D \leq d_{T}(X, Y) \leq \bar{d}(X, Y)+D, \\
\delta_{L}(X, Y)-D \leq d_{T}(X, Y) \leq \delta_{L}(X, Y)+D
\end{gathered}
$$

Proof We first prove the right hand side of Inequality (10).

Consider the function

$$
(X, \gamma) \mapsto \frac{E_{X}(\gamma)}{l_{X}^{2}(\gamma)},
$$

defined for $X$ in the $\varepsilon_{0}$-relative $\epsilon$-thick part of $\mathcal{T}(S)$ and $\gamma$ in $\mathcal{P} \mathcal{M} \mathcal{F}(S)$. From the definition of the length of a measured lamination (see [8]), we have the homogeneity property $E_{X}(t \gamma)=t^{2} E_{X}(\gamma)$ and $l_{X}^{2}(t \gamma)=t^{2} l_{X}^{2}(\gamma)$ for all $t>0$. Since $\mathcal{P \mathcal { N }} \mathcal{F}(S)$ is compact and since the image of the $\varepsilon_{0}$-relative $\epsilon$-thick part of $\mathcal{T}(S)$ in $\mathcal{N}(S)$ is compact (Theorem 4.1), the values taken by the function (13) are uniformly bounded from above, that is, there exists a positive constant $C$ which depends only on $\epsilon$ and $\varepsilon_{0}$ such that

$$
\frac{E_{X}(\gamma)}{l_{X}^{2}(\gamma)} \leq C .
$$

It follows from (1) and (14) that for any $X, Y$ in the $\varepsilon_{0}$-relative $\epsilon$-thick part of $\mathcal{T}(S)$, we have

$$
\frac{E_{Y}(\gamma)}{E_{X}(\gamma)} \leq \frac{2 \pi|\chi(S)|}{l_{X}^{2}(\gamma)} C l_{Y}^{2}(\gamma) .
$$

Therefore,

$$
\begin{aligned}
d_{T}(X, Y)=\frac{1}{2} \log \sup _{\gamma \in \mathcal{C}(S)} \frac{E_{Y}(\gamma)}{E_{X}(\gamma)} & \leq \frac{1}{2} \log \sup _{\gamma \in \mathcal{C}(S)} \frac{l_{Y}^{2}(\gamma)}{l_{X}^{2}(\gamma)}+\frac{1}{2} \log (2 \pi|\chi(S)| C) \\
& =\log \sup _{\gamma \in \mathcal{C}(S)} \frac{l_{Y}(\gamma)}{l_{X}(\gamma)}+\frac{1}{2} \log (2 \pi|\chi(S)| C) \\
& =d(X, Y)+\frac{1}{2} \log (2 \pi|\chi(S)| C) .
\end{aligned}
$$

From this, we obtain the right hand side inequality in (10).

The proof of the right hand side inequality in (11) is similar.

We can use (1) and (14) again to show that

$$
\frac{E_{Y}(\gamma)}{E_{X}(\gamma)} \geq \frac{l_{X}^{2}(\gamma)}{2 \pi|\chi(S)| C l_{Y}^{2}(\gamma)},
$$

which gives

$$
d_{T}(X, Y) \geq 2 \bar{d}(X, Y)-D \geq \bar{d}(X, Y)-D
$$


for some $D$ depending on $\varepsilon_{0}$ and $\epsilon$. Since $d_{T}$ is symmetric, we also have

$$
d_{T}(X, Y) \geq d(X, Y)-D \text {. }
$$

Inequalities (12) follow from the two previous ones and from the definition $\delta_{L}(X, Y)=\max (d(X, Y), \bar{d}(X, Y))$.

Theorem 6.3 gives in particular a comparison between the functions $d$ and $d_{T}$ for pairs of points in an $\varepsilon_{0}$-relative $\epsilon$-thick part of Teichmüller space. The following theorem gives information on pairs of points that are not necessarily in an $\varepsilon_{0}$-relative $\epsilon$-thick part of the space.

Theorem 6.4 Let $S$ be a surface of topologically finite type and $\left(X_{n}\right)_{n \in \mathbb{N}}$ be a sequence of elements in $\mathcal{T}(S)$. Then,

$$
\lim _{n \rightarrow \infty} d_{T}\left(X_{n}, X_{0}\right)=\infty \Longleftrightarrow \lim _{n \rightarrow \infty} d\left(X_{n}, X_{0}\right)=\infty
$$

Proof Theorem 6.1 implies that if $\lim _{n \rightarrow \infty} d\left(X_{n}, X_{0}\right)=\infty$, then we also have $\lim _{n \rightarrow \infty} d_{T}\left(X_{n}, X_{0}\right)=\infty$.

For the converse, suppose that $\lim _{n \rightarrow \infty} d_{T}\left(X_{n}, X_{0}\right)=\infty$. First, consider the following two special cases:

(1) The sequence $\left(X_{n}\right)_{n \in \mathbb{N}}$ stays in some $\varepsilon_{0}$-relative $\epsilon$-thick of $\mathcal{T}(S)$ for some $\varepsilon_{0} \geq$ $\epsilon>0$. In this case, by Theorem 6.3 (10), $\lim _{n \rightarrow \infty} d\left(X_{n}, X_{0}\right)=\infty$.

(2) The sequence $\left(X_{n}\right)_{n \in \mathbb{N}}$ leaves any $\varepsilon_{0}$-relative $\epsilon$-thick part of $\mathcal{T}(S)$. In this case it follows from the definition of $d$ that $\lim _{n \rightarrow \infty} d\left(X_{n}, X_{0}\right)=\infty$.

Now we discuss the general case. We reason by contradiction. Suppose that the sequence $d\left(X_{n}, X_{0}\right)$ does not go to infinity as $n$ goes to infinity. Then there exists a subsequence $\left(X_{n_{k}}\right)_{k \in \mathbb{N}}$ of $\left(X_{n}\right)$ such that the set $\left\{d\left(X_{n_{k}}, X_{0}\right), k \in \mathbb{N}\right\}$ is bounded. From the second special case above, if the subsequence $\left(X_{n_{k}}\right)$ leaves any $\varepsilon_{0}$-relative $\epsilon$-thick part of $\mathcal{T}(S)$, we get a contradiction. Therefore, up to taking a subsequence again, we can assume that there exists an $\varepsilon_{0}$-relative $\epsilon$-thick part of $\mathcal{T}(S)$ which contains the sequence of $\left(X_{n_{k}}\right)$. But the first case above shows that this yields a contradiction.

Theorem 6.5 Let $S$ be a surface of topologically finite type and let $\left(X_{n}\right)_{n \geq 0}$ be a sequence of elements in $\mathcal{T}(S)$. Then,

$$
\lim _{n \rightarrow \infty} d_{T}\left(X_{n}, X_{0}\right)=0 \text { if and only if } \lim _{n \rightarrow \infty} \delta_{L}\left(X_{n}, X_{0}\right)=0
$$

Proof We know that, for any $X, Y \in \mathcal{T}(S), d_{T}(X, Y)=d_{T}\left(X^{d}, Y^{d}\right)$ and $\delta_{L}(X, Y)=$ $\delta_{L}\left(X^{d}, Y^{d}\right)$. By the topological equivalence proved in [10,15], we have

$$
\lim _{n \rightarrow \infty} d_{T}\left(X_{n}^{d}, X_{0}^{d}\right)=0 \text { if and only if } \lim _{n \rightarrow \infty} \delta_{L}\left(X_{n}^{d}, X_{0}^{d}\right)=0
$$

This proves the theorem. 
We proved in [9] that for any surface $S$ of topologically finite type, $d, \bar{d}, d_{L}$ and $\delta_{L}$ induce the same topology on $\mathcal{T}(S)$. Putting this result with Theorem 6.5 we get the following

Theorem 6.6 Let $S$ be a surface of topologically finite type. The weak metrics $d, \bar{d}$, and the metrics $d_{L}, \delta_{L}$ and $d_{T}$ induce the same topology on $\mathcal{T}(S)$.

Open Access This article is distributed under the terms of the Creative Commons Attribution Noncommercial License which permits any noncommercial use, distribution, and reproduction in any medium, provided the original author(s) and source are credited.

\section{References}

1. Abikoff, W.: The real analytic theory of Teichmüller space. Lecture Notes in Mathematics 820. Springer (1980)

2. Ahlfors, L.V., Beurling, A.: Conformal Invariants. In: Ahlfors, L.V., Collected papers, vol. 1 (19291955), pp. 435-437. Birkhäuser, Boston (1982)

3. Bers, L.: Nielsen extensions of Riemann surfaces. Ann. Acad. Sci. Fenn. Ser. AI. Math. 2, 29-34 (1976)

4. Choi, Y., Rafi, K.: Comparison between Teichmüller and Lipschitz metrics. J.Lond. Math. Soc. 76, 739756 (2007)

5. Fathi, A., Laudenbach, F., Poénaru, V.: Travaux de Thurston sur les surfaces. Astérisque, 66-67 (1979)

6. Hubbard, J.H.: Teichmüller theory, vol. 1. Matrix Editions, Ithaca (2006)

7. Hubbard, J.H., Masur, H.: Quadratic differentials and measured foliations. Acta Math. 142, 221274 (1979)

8. Kerckhoff, S.: The asymptotic geometry of Teichmüller space. Topology 19, 23-41 (1980)

9. Liu, L., Papadopoulos, A., Su, W., Théret, G.: On length spectrum metrics and weak metrics on Teichmüller spaces of surfaces with boundary, preprint, arXiv:0903.0744v1 (2009)

10. Liu, L.: On the length spectrums of non-compact Riemann surfaces. Ann. Acad. Sci. Fenn. Math. 24, 11-22 (1999)

11. Liu, L.: On the metrics of length spectrum in Teichmüller space. Chinese J. Cont. Math. 22(1), 23-34 (2001)

12. Liu, L., Sun, Z., Wei, H.: Topological equivalence of metrics in Teichmüller space. Ann. Acad. Sci. Fenn. Math. 33(1), 159-170 (2008)

13. Maskit, B.: Comparison of hyperbolic length and extremal length. Ann. Acad. Sci. Fenn. Ser. AI Math. 10, 381-386 (1985)

14. Mumford, D.: A remark on Mahler's compactness theorem. Proc. Am. Math. Soc. 28, 289-294 (1971)

15. Papadopoulos, A., Théret, G.: On the topology defined by Thurston's asymmetric metric. Math. Proc. Camb. Phil. Soc. 142, 487-496 (2007)

16. Parlier, H.: Lengths of geodesics on Riemann surfaces with boundary. Ann. Acad. Sci. Fenn. Math. 30(2), 227-236 (2005)

17. Sorvali, T.: On Teichmüller spaces of tori. Ann. Acad. Sci. Fenn. Ser. AI Math. 1, 7-11 (1975)

18. Strebel, K.: Quadratic differentials. Ergebnisse der Mathematik und ihrer Grenzgebiete 3, Band 5. Springer, Berlin (1984)

19. Thurston, W.P.: The geometry and topology of Three-manifolds, Mimeographed notes, Princeton University. Available on the web at: http://www.msri.org/publications/books/gt3m/ (1976)

20. Thurston, W.: Minimal stretch maps between hyperbolic surfaces, preprint, 1086, Arxiv:math GT/9801039

21. Wolpert, S.: The length spectra as moduli for compact Riemann surfaces. Ann. Math. 109, 323-351 (1979) 